\title{
Carotenoid metabolism during bilberry (Vaccinium myrtillus L.) fruit development under different light conditions is regulated by biosynthesis and degradation
}

Katja Karppinen ${ }^{1,2}$, Laura Zoratti ${ }^{1}$, Marian Sarala ${ }^{1}$, Elisabete Carvalho ${ }^{3}$, Jenni Hirsimäki ${ }^{1}$, Helmi Mentula ${ }^{1}$, Stefan Martens ${ }^{3}$, Hely Häggman ${ }^{1}$ and Laura Jaakola ${ }^{2,4^{*}}$

\begin{abstract}
Background: Carotenoids are important pigments and precursors for central signaling molecules associated in fruit development and ripening. Carotenoid metabolism has been studied especially in the climacteric tomato fruit but the content of carotenoids and the regulation of their metabolism have been shown to be highly variable between fruit species. Non-climacteric berries of the genus Vaccinium are among the best natural sources of health-beneficial flavonoids but not studied previously for carotenoid biosynthesis.

Results: In this study, carotenoid biosynthetic genes, PSY, PDS, ZDS, CRTISO, LCYB, LCYE, BCH and CYP450-BCH, as well as a carotenoid cleavage dioxygenase CCD1 were identified from bilberry ( $V$. myrtillus L.) fruit and their expression was studied along with carotenoid composition during fruit development under different photoperiod and light quality conditions. Bilberry was found to be a good source of carotenoids among fruits and berries. The most abundant carotenoids throughout the berry development were lutein and $\beta$-carotene, which were accompanied by lower amounts of 9Z- $\beta$ carotene, violaxanthin, neoxanthin, zeaxanthin, antheraxanthin and $\beta$-cryptoxanthin. The expression patterns of the biosynthetic genes in ripening fruits indicated a metabolic flux towards $\beta$-branch of the carotenoid pathway. However, the carotenoid levels decreased in both the $\beta$-branch and $\varepsilon, \beta$-branch towards bilberry fruit ripening along with increased VmCCD1 expression, similarly to VmNCED1, indicating enzymatic carotenoid cleavage and degradation. Intense white light conditions increased the expression of the carotenoid biosynthetic genes but also the expression of the cleavage genes VmCCD1 and VmNCED1, especially in unripe fruits. Instead, mature bilberry fruits responded specifically to red/far-red light wavelengths by inducing the expression of both the carotenoid biosynthetic and the cleavage genes indicating tissue and developmental stage specific regulation of apocarotenoid formation by light quality.
\end{abstract}

Conclusions: This is the first report of carotenoid biosynthesis in Vaccinium berries. Our results indicate that both transcriptional regulation of the key biosynthetic genes and the enzymatic degradation of the produced carotenoids to apocarotenoids have significant roles in the determination of the carotenoid content and have overall effect on the metabolism during the bilberry fruit ripening.

Keywords: Vaccinium, Carotenoid biosynthesis, Berry ripening, Lutein, Beta-carotene, Gene expression, Red light

\footnotetext{
*Correspondence: laura.jaakola@uit.no

${ }^{2}$ Climate laboratory Holt, Department of Arctic and Marine Biology, UiT the

Arctic University of Norway, NO-9037 Tromsø, Norway

${ }^{4} \mathrm{NIBIO}$, Norwegian Institute of Bioeconomy Research, P.O. Box 115 NO-1431

Ås, Norway

Full list of author information is available at the end of the article
} 


\section{Background}

Fruits and berries are important components of the human diet providing a source of many nutritive and bioactive compounds such as carotenoids [1, 2]. The bright red and yellow carotenoids give color for many flowers and fruits for attracting pollinators and seed dispersers. Carotenoids play also other essential roles in plants by being involved in photosystem assembly, light-harvesting and photoprotection [3]. Moreover, they serve as precursors for important carotenoid cleavage products called apocarotenoids, which include phytohormone abscisic acid (ABA), strigolactones and volatile flavor compounds [4-6]. Humans cannot biosynthesize carotenoids and, therefore, these essential compounds need to be acquired from the diet. Carotenoids are essential for humans as precursors of vitamin A but they also provide other health-benefits due to their antioxidant properties. Consumption of carotenoid rich food can enhance immune system and certain carotenoids have been shown to exert protective effects against cardiovascular diseases, certain types of cancers as well as degenerative diseases [7, 8]. Especially lutein and zeaxanthin have an ability to slow down age-related damage to the eye retina [9].

Due to the importance of carotenoids to plants and humans, carotenoid biosynthetic pathway in plants (Fig. 1a) is well established and it takes place in plastids by nuclear-encoded enzymes $[3,6,10]$. The first committed step in the carotenoid biosynthesis, the condensation of two molecules of geranylgeranyl diphosphate (GGPP) to phytoene by phytoene synthase (PSY), has in many plant systems been reported as the rate-limiting step controlling the metabolic flux to carotenoid biosynthesis $[3,5]$. By a series of reactions, phytoene is desaturated to lycopene involving the action of phytoene

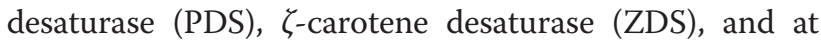
least two isomerases, including carotenoid isomerase (CRTISO) [3]. In some fruits, such as tomato (Solanum lycopersicum L.), the red lycopene is the major accumulating carotenoid compound. In the branching point of the carotenoid pathway, lycopene can be further cyclized by lycopene cyclases, lycopene $\beta$-cyclase (LCYB) and lycopene $\varepsilon$-cyclase (LCYE), to form either $\alpha$-carotene or $\beta$-carotene. In the $\varepsilon, \beta$-branch, biosynthesis of lutein from $\alpha$-carotene requires sequential action of two separate carotenoid hydroxylases belonging to the cytochrome P450 family, $\beta$-ring hydroxylase $(\mathrm{CYP} 450-\mathrm{BCH})$ and $\varepsilon$ ring hydroxylase (CYP450-ECH) [10]. In the $\beta$-branch of the carotenoid pathway, hydroxylation of $\beta$-carotene by $\beta$-carotene hydroxylase $(\mathrm{BCH})$ produces zeaxanthin via $\beta$-cryptoxanthin for the xanthophyll cycle. Epoxidation of the zeaxanthin in the ABA biosynthetic pathway leads to the formation of violaxanthin and neoxanthin, which can be further cleaved by 9-cis-

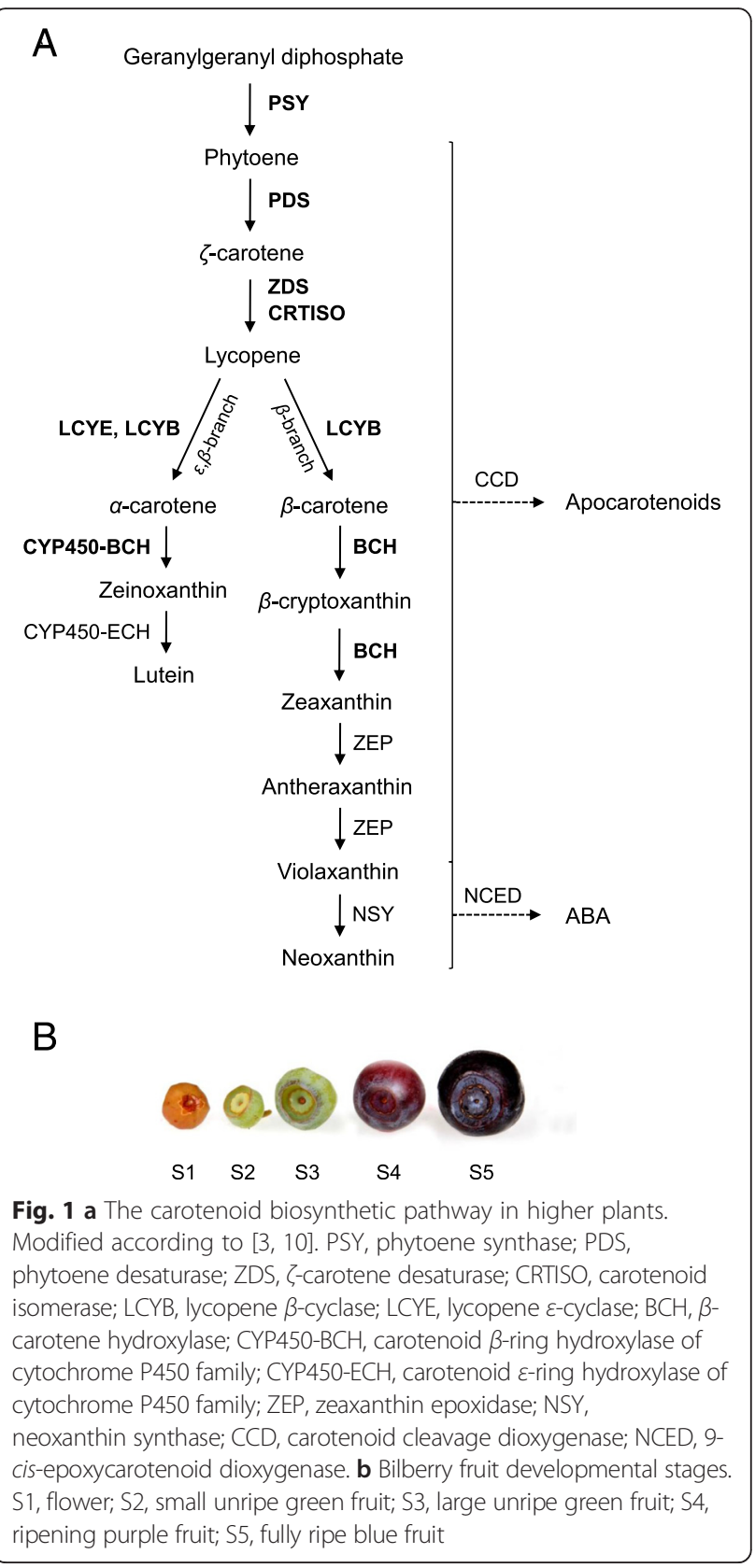

epoxycarotenoid dioxygenase (NCED) to produce plant hormone ABA $[3,6,11]$.

Previous studies on carotenoid biosynthesis have evidenced that in many fruits, the coordinated transcriptional regulation of the carotenoid biosynthetic genes, especially PSY, PDS and lycopene cyclases, is the key determinant of carotenoid profile [5, 12-15]. For example, tomato carotenoid composition coincides well with the up-regulation of the early carotenoid biosynthetic genes and the down-regulation of the downstream genes of the accumulating carotenoids [16]. However, beyond the transcript level, carotenoid metabolism is shown to be regulated in a much more complex manner by an 
elaborate coordinated regulatory network associated in fruit development and ripening [6]. Much of the knowledge of fruit carotenogenesis has been achieved from studies with tomato, a model system of the climacteric fruit ripening $[5,6]$, while the regulatory mechanisms affecting to the fruit ripening and carotenoid composition has been shown to vary in different fruit species and compared to the species showing non-climacteric fruit ripening [17]. In addition to the transcriptional regulation, factors such as post-transcriptional regulation, chromoplast biogenesis, epigenetic mechanisms and enzymatic degradation of carotenoids to apocarotenoids by the carotenoid cleavage dioxygenases (CCDs) can have a profound role on the fruit carotenoid metabolism $[3,5,6]$. In fact, some apocarotenoid compounds are recognized as important signaling, aroma, flavor and pigment components in fruits and berries [18-20].

In addition to the genetic factors, environmental factors, such as quantity of light, are recognized as regulators of carotenoid biosynthesis in chloroplasts. Although differential mechanisms are involved, there are few reports indicating the regulation of carotenogenesis in chromoplasts of fruits by light quality. In tomato and Citrus fruits, especially red light wavelengths have been demonstrated to increase carotenoid biosynthesis and accumulation [21, 22].

Bilberry (Vaccinium myrtillus L., Fig. 1b) is one of the most abundant wild berries in the Northern Europe recognized for its exceptionally high anthocyanin content indicated by the deep blue colour of ripe fruits [23]. Besides flavonoids, these berries are potential sources of other health-beneficial compounds and previously we have shown them to be a moderate source of vitamin C [24]. Although carotenoid biosynthesis has been studied in various fruit producing species, the reports of the carotenoid metabolism in non-climacteric Vaccinium fruits are scarce. Previous measurements have shown that lutein and $\beta$-carotene are the major carotenoids in ripe bilberry and blueberry ( $V$. corymbosum L.) fruits [25-28] but studies on the carotenoid biosynthesis at different phases of fruit development and ripening are lacking. In the present work, we aimed to measure in detail the levels of carotenoids and analyze their biosynthesis and degradation during bilberry fruit development (Fig. 1b). For this purpose, eight carotenoid biosynthetic genes (VmPSY, VmPDS, VmZDS, VmCRTISO, $V m L C Y B, V m L C Y E, V m B C H$ and $V m C Y P 450-B C H)$ as well as a carotenoid cleavage gene $V m C C D 1$ were cloned, and their expression patterns were determined in the bilberry fruits at different stages of development and ripening. In addition, the effect of various light conditions on the carotenoid metabolism in unripe and mature berries was studied.

\section{Results}

\section{Identification of carotenoid biosynthetic genes and CCD1} in bilberry

In order to examine the bilberry fruit development related carotenoid biosynthesis at a molecular level, sequences of the genes phytoene synthase (VmPSY), phytoene desaturase (VmPDS), $\zeta$-carotene desaturase $(V m Z D S)$, carotenoid isomerase (VmCRTISO), lycopene $\beta$-cyclase $(V m L C Y B)$, lycopene $\varepsilon$-cyclase $(V m L C Y E), \beta$ carotene hydroxylase $(\mathrm{VmBCH})$ and carotenoid $\beta$-ring hydroxylase of cytochrome $\mathrm{P} 450$ family (VmCYP450$B C H)$ were isolated. All the isolated sequences showed high identities to the corresponding sequences reported previously in the fruit carotenoid biosynthesis in other species (Table 1). Also the sequence of the isolated carotenoid cleavage gene $V m C C D 1$ of bilberry showed a high identity to the CCD1 class enzymes associated with carotenoid cleavage in other fruit species (Table 1). For example, $V m C C D 1$ is $89 \%$ identical at the amino acid level to the $V v C C D 1$ of grape (Vitis vinifera L.) berry, which is implicated in the cleavage of zeaxanthin and lutein for the formation of $\mathrm{C}_{13^{-}}$and $\mathrm{C}_{14}$-apocarotenoids [29]. All the obtained sequences from bilberry were deposited to the GenBank database (Table 1).

\section{Expression of carotenoid biosynthetic genes and CCD1 during bilberry fruit development}

Expression of the carotenoid biosynthetic genes and the cleavage gene $V m C C D 1$ was analyzed in bilberry fruit at five different developmental stages (Fig. 1b) by qRTPCR. All the eight examined biosynthetic genes were expressed at detectable levels throughout bilberry fruit development but with variable expression patterns (Fig. 2). The expression of the early biosynthetic genes VmPSY, VmPDS and VmCRTISO showed highly similar patterns with relatively low expression at the early stages of fruit development but approximately four-, eight- and six-fold increment, respectively, at the onset of fruit ripening (S4). Their expression was relatively high also in ripe fruit (S5). The expression of $V m Z D S$ was high in flowers (S1) decreasing at the beginning of fruit development and increasing again towards the ripe fruit. The expression of $V m L C Y B$, which has a role both in $\varepsilon, \beta$ and $\beta$-branches of the carotenoid pathway, resembled that of VmPSY, VmPDS and VmCRTISO showing a fivefold increment in its expression at the fruit ripening (S4) while the expression of $\mathrm{VmBCH}$, a $\beta$-branch gene, showed increase already at the green fruit stage (S3). The transcript levels of the genes specific to $\varepsilon, \beta$-branch, $V m L C Y E$ and $V m C Y P 450-B C H$, were relatively high at the stage of flowering (S1) as well as at the green stage (S3) but were down-regulated at the onset of fruit ripening (S4). The expression of the VmLCYE was upregulated again in ripe berries (S5). The expression of 
Table 1 The identity of carotenoid biosynthetic and cleavage genes of bilberry compared with other fruit species

\begin{tabular}{|c|c|c|c|}
\hline Gene & GenBank accession no. & Clone size (bp) & Identity at amino acid level (\%) \\
\hline \multirow[t]{3}{*}{ VMPSY } & KR706538 & 806 & 93 (Actinidia deliciosa, ACO53104) \\
\hline & & & 92 (Diospyros kaki, ACM44688) \\
\hline & & & 89 (Citrus sinensis, ABB72444) \\
\hline \multirow[t]{3}{*}{ VmPDS } & KR706539 & 828 & 92 (Diospyros kaki, ACY78343) \\
\hline & & & 91 (Vitis vinifera, AFP28796) \\
\hline & & & 90 (Citrus sinensis, ABB72445) \\
\hline \multirow[t]{3}{*}{$V m Z D S$} & KR706540 & 553 & 86 (Citrus sinensis, NP_001275793) \\
\hline & & & 85 (Vitis vinifera, AFP28797) \\
\hline & & & 85 (Lycium barbarum, AIX87496) \\
\hline \multirow[t]{3}{*}{ VMCRTISO } & KR706541 & 369 & 92 (Lycium barbarum, AIX87497) \\
\hline & & & 89 (Solanum lycopersicum, AAL91366) \\
\hline & & & 89 (Citrus unshiu, AlG20207) \\
\hline \multirow[t]{3}{*}{$V m L C Y B$} & KR706542 & 487 & 93 (Capsicum annuum, ADH04271) \\
\hline & & & 93 (Vitis vinifera, AFP28799) \\
\hline & & & 91 (Diospyros kaki, ACR25158) \\
\hline \multirow[t]{3}{*}{$V m L C Y E$} & KR706543 & 529 & 87 (Diospyros kaki, BAE94036) \\
\hline & & & 82 (Coffea canephora, ABC87738) \\
\hline & & & 81 (Citrus limon, BAD07293) \\
\hline \multirow[t]{3}{*}{$\mathrm{VmBCH}$} & KR706544 & 783 & 78 (Capsicum annuum, CAA70888) \\
\hline & & & 77 (Diospyros kaki, ACN86365) \\
\hline & & & 76 (Coffea arabica, ABA43903) \\
\hline \multirow[t]{2}{*}{ VmCYP450-BCH } & KR706545 & 715 & 82 (Solanum lycopersicum CYP97A29, ACJ25969) \\
\hline & & & 79 (Lycium ruthenicum CYP97A29, AIX87527) \\
\hline \multirow[t]{3}{*}{ VmCCD1 } & KR706546 & 909 & 89 (Vitis vinifera, AAX48772) \\
\hline & & & 87 (Citrus sinensis, BAE92958) \\
\hline & & & 86 (Coffea arabica, ABA43904) \\
\hline
\end{tabular}

the carotenoid cleavage gene $V m C C D 1$ was found to increase during the berry development (Fig. 3), especially at the stage of fruit ripening (S4), resembling the expression patterns of VmPSY, VmPDS, VmCRTISO and $V m L C Y B$.

\section{Carotenoid content during bilberry fruit development}

The carotenoid composition and concentrations in the bilberry fruit were analyzed in detail at the five developmental stages (Fig. 1b) by HPLC-DAD revealing the presence of carotenes and xanthophylls. Among carotenes, $\beta$-carotene and smaller amount of 9Z- $\beta$-carotene were detected whereas xanthophylls detected in bilberry fruit included lutein, zeaxanthin, antheraxanthin, violaxanthin, neoxanthin (Fig. 4) and traces amounts of $\beta$-cryptoxanthin. No lycopene was detected in bilberry fruit.

Lutein was found to be the most abundant carotenoid in bilberry fruit at every developmental stage followed by $\beta$-carotene (Fig. 4). The levels of both lutein and $\beta$ carotene were highest in small unripe green fruit (S2) and the amounts decreased during the fruit development although there was a slight increase in the lutein concentration at the end of fruit ripening. In ripe fruit, the final concentrations of lutein and $\beta$-carotene were $1476 \mu \mathrm{g} 100 \mathrm{~g}^{-1} \mathrm{DW}\left(184 \mu \mathrm{g} 100 \mathrm{~g}^{-1} \mathrm{FW}\right)$ and $380 \mu \mathrm{g}$ $100 \mathrm{~g}^{-1} \mathrm{DW}\left(47 \mu \mathrm{g} 100 \mathrm{~g}^{-1} \mathrm{FW}\right)$, respectively. Also the levels of $9 \mathrm{Z}-\beta$-carotene, zeaxanthin, antheraxanthin, violaxanthin and neoxanthin showed a decreasing trend during the bilberry fruit development (Fig. 4). The highest levels of these compounds were detected in small unripe green fruit (S2) with the exception of zeaxanthin concentration being the highest in flowers (S1). The total carotenoid content in the ripe bilberry fruit was $2872 \mu \mathrm{g} 100 \mathrm{~g}^{-1} \mathrm{DW}\left(359 \mu \mathrm{g} 100 \mathrm{~g}^{-1} \mathrm{FW}\right)$ of which approximately $64 \%$ constituted of lutein and $\beta$-carotene.

\section{Expression of carotenoid biosynthetic genes in bilberry fruit at different light conditions}

Expression of the carotenoid biosynthetic genes was evaluated after treatment of unripe (S3) and ripe (S5) 


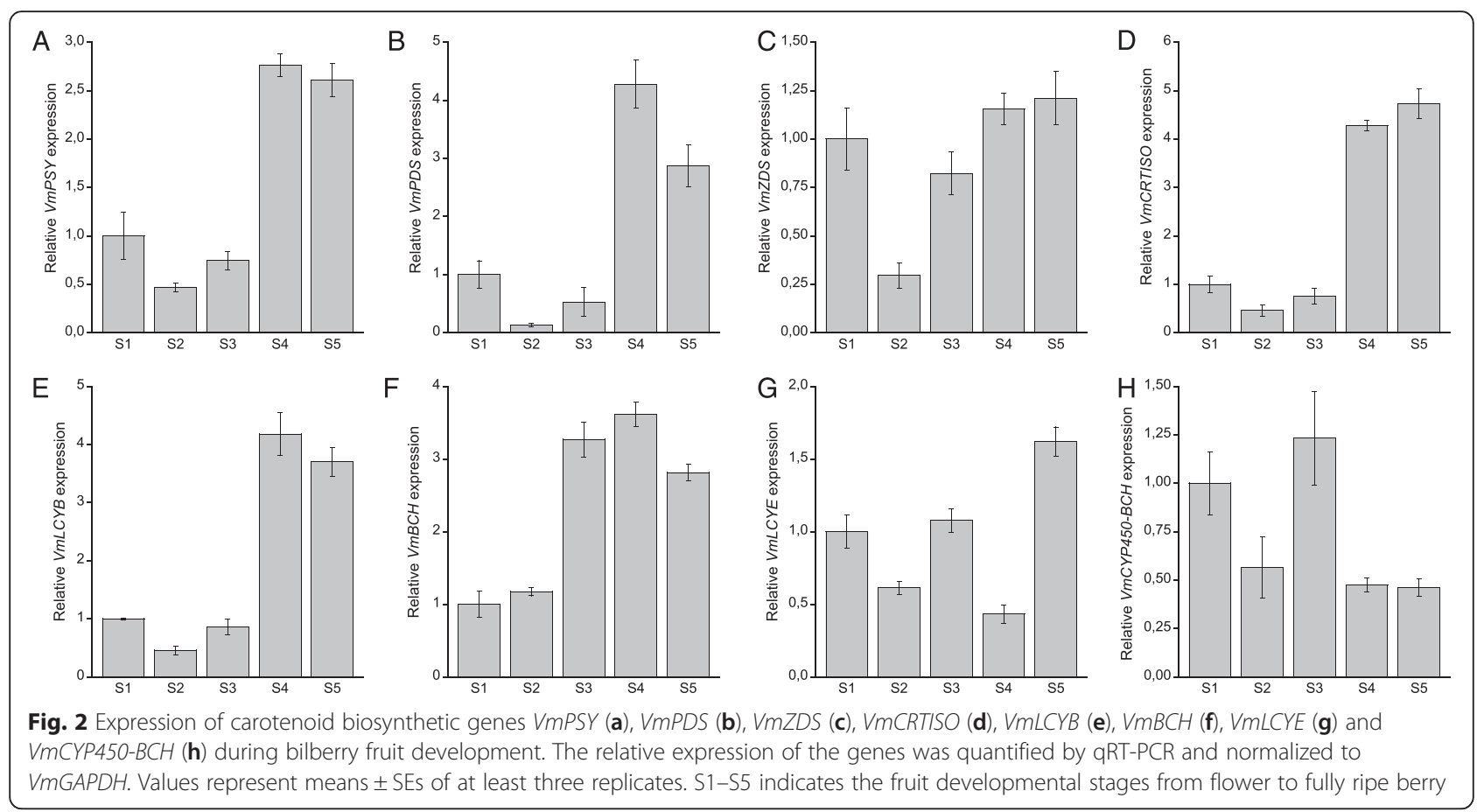

bilberry fruits with white light. In both unripe and ripe berries, photoperiodic $(16 / 8 \mathrm{~h})$ white light significantly induced the expression of VmPSY, VmPDS, VmLCYB and $V m L C Y E$ compared to the control berries kept in darkness (Fig. 5). The expression of the other biosynthetic genes, VmZDS, VmCRTISO, $V m B C H$ and VmCYP450-

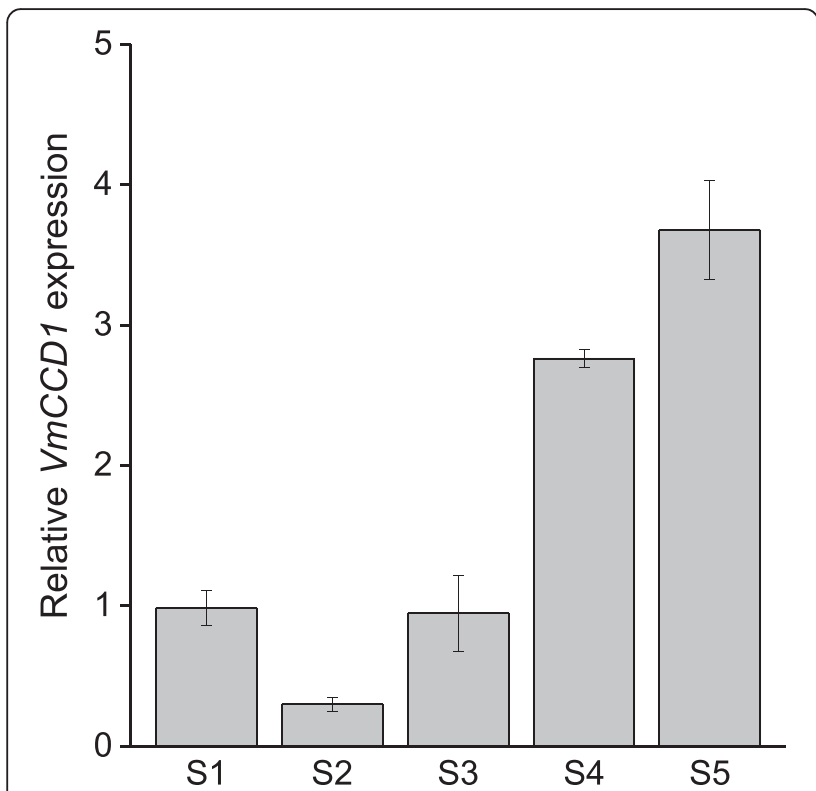

Fig. 3 Expression of VmCCD1 gene during bilberry fruit

development. The relative expression of the gene was quantified by qRT-PCR and normalized to VmGAPDH. Values represent means \pm SEs of at least three replicates. S1-S5 indicates the fruit developmental stages from flower to fully ripe berry
$B C H$, did not show a marked response to the photoperiodic white light treatment, although the increase in the $V m C Y P 450-B C H$ expression in ripe fruit after $60 \mathrm{~h}$ treatment was significant.

The effect of photoperiod and light quality on bilberry fruit carotenoid biosynthesis was further investigated by a $60 \mathrm{~h}$-treatment with continuous $(24 \mathrm{~h})$ white light and photoperiodic $(16 / 8 \mathrm{~h})$ white light with elevated red/farred wavelengths. In unripe berries, the treatment with continuous white light had a significant increasing effect on the expression of VmPSY, VmPDS, VmCRTISO, $V m L C Y B$ and $V m L C Y E$ compared with the control berries kept in darkness as well as the berries grown under photoperiodic white light (Fig. 6). Instead, the expression of $V m Z D S, V m B C H$ and $V m C Y P 450-B C H$ in unripe berries was not elevated by the continuous white light treatment compared to the photoperiodic white light treatment. The additional red/far-red light wavelengths did not have a significant elevating effect on the gene expression in unripe berries compared to the photoperiodic white light treatment, although a slight increase in the expression of VmPDS, VmCRTISO and VmLCYB was observed. In ripe berries, light had differential effect on the expression of the carotenoid biosynthetic genes compared to unripe berries and, generally, continuous and photoperiodic white light treatments had no or only a slight inducing effect on the expression of the carotenoid biosynthetic genes after $60 \mathrm{~h}$ treatment (Fig. 6). Instead, the expression of VmPSY, VmPDS, VmCRTISO, VmLCYB and $V m L C Y E$ was increased in ripe berries grown under elevated red/far-red light wavelengths in the $16 / 8 \mathrm{~h}$ 


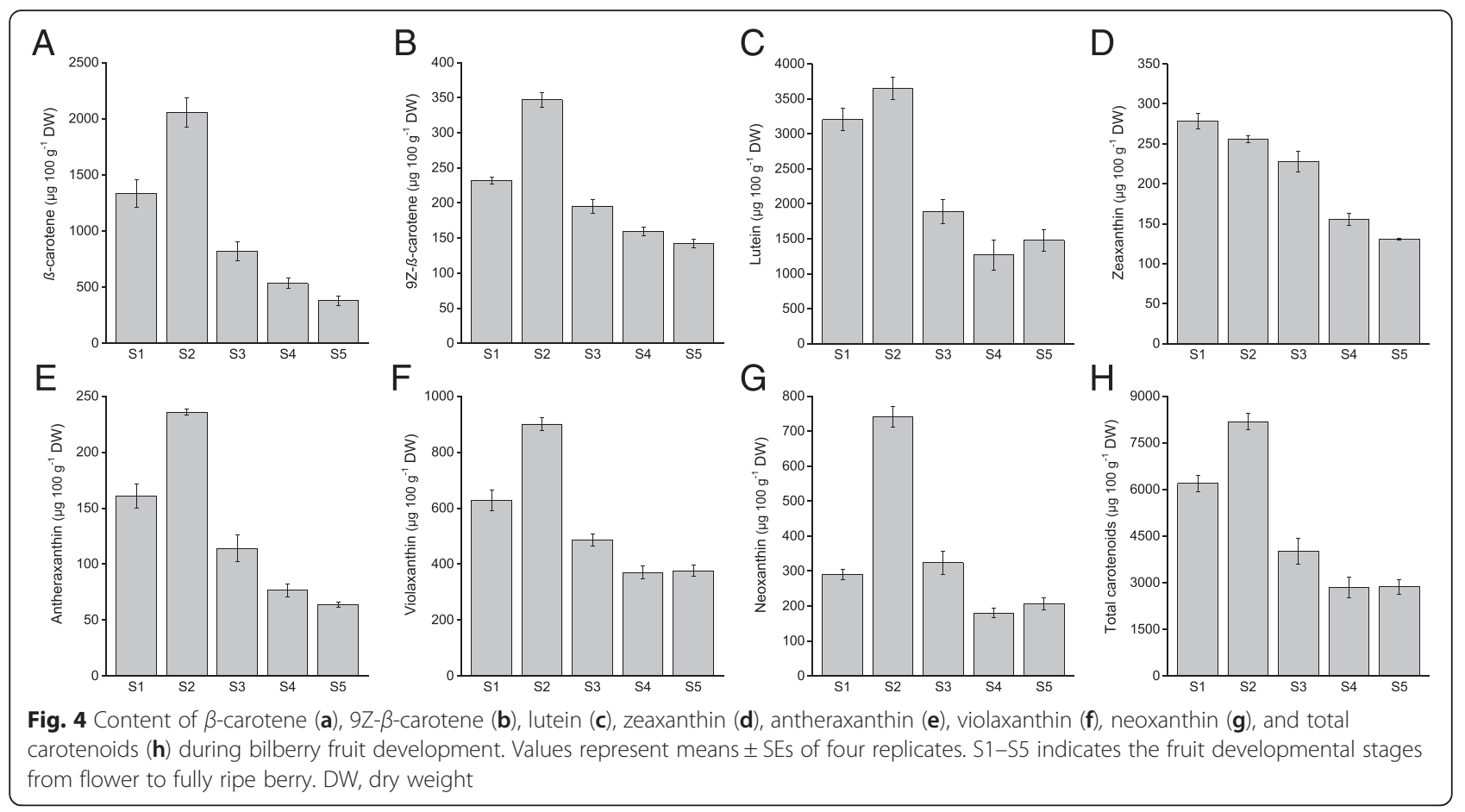

photoperiod compared to all the other treatments. The expression of $V m Z D S, V m B C H$ and $V m C Y P 450-B C H$ in ripe berries did not show a marked response to the treatment.

\section{Expression of carotenoid cleavage genes in bilberry fruit at different light conditions}

Expression of the carotenoid cleavage genes, $V m C C D 1$ and the previously isolated $V m N C E D 1$, the key gene in bilberry ABA biosynthesis [11], was measured after the treatment of unripe and ripe bilberry fruits with different light conditions. The results show that the expression of both of these cleavage genes could be induced by a $16 / 8 \mathrm{~h}$ photoperiodic white light treatment (Fig. 7). Continuous white light exposure for $60 \mathrm{~h}$ had a significant inducing effect on the expression of the cleavage genes in unripe berries compared with the control berries kept in darkness as well as the berries grown under photoperiodic white light (Fig. 8). Elevation of red/far-red light wavelengths in photoperiodic white light had a significant increasing effect on the expression of $V m C C D 1$ and it also slightly increased the expression of $V m N C E D 1$ in unripe berries. In ripe berries, the $60 \mathrm{~h}$ white light treatments did not significantly affect the gene expression but instead red/far-red light wavelengths had a significant inducing effect on the expression of both of the cleavage genes.

\section{Carotenoid content of bilberry fruit at different light conditions}

The effect of different light conditions on the accumulation of carotenoids was analyzed after five days of the initiation of each light treatment. The metabolic profile of unripe berries showed a slight increment in the accumulation of both $\beta$-branch and $\varepsilon, \beta$-branch carotenoids after white light exposure (Table 2). The increase in $\beta$ carotene concentration was significant after five days treatment with continuous white light compared to the dark grown control berries. On the contrary, in ripe berries, all the light treatments led to the decreased content of carotenoids compared to the berries grown in dark (Table 2).

\section{Discussion}

\section{Bilberries are good source of carotenoids among fruits} and berries

Carotenoids have been shown to accumulate in chromoplasts of fruits with variable profiles and concentrations between species and even between close cultivars [30-33]. Bilberry fruits accumulate high amounts of anthocyanin pigments during the ripening. The biosynthesis and content of carotenoids during the fruit development and ripening has not been evaluated earlier in the genus Vaccinium. The results of the current study are in agreement with the earlier measurements that have indicated lutein and $\beta$-carotene as the main carotenoids in ripe bilberry fruits [25-28]. Our study demonstrates that the total carotenoid content in ripe bilberries is higher than described for example in ripe fruits of raspberry $(R u$ bus idaeus L.), grape berry, strawberry (Fragaria $\times$ ananassa) or commercial apple (Malus $\times$ domestica) cultivars [25, 31, 34, 35] indicating that among fruits 
A

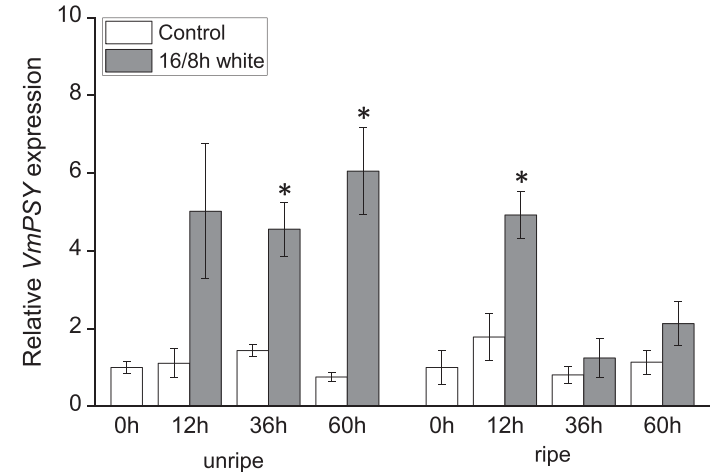

C

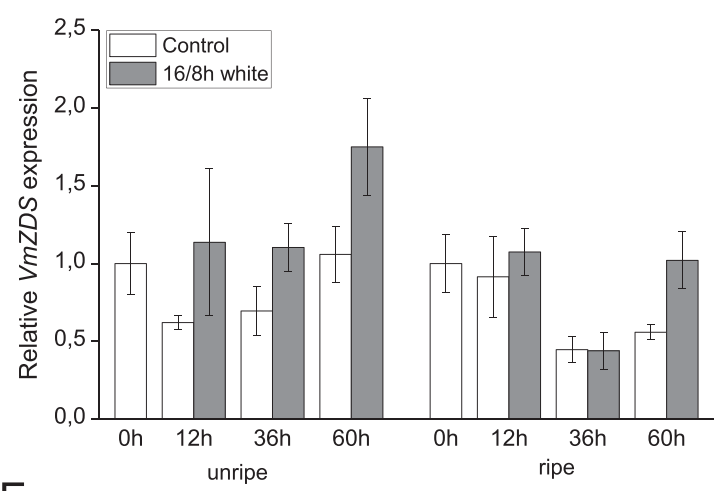

E

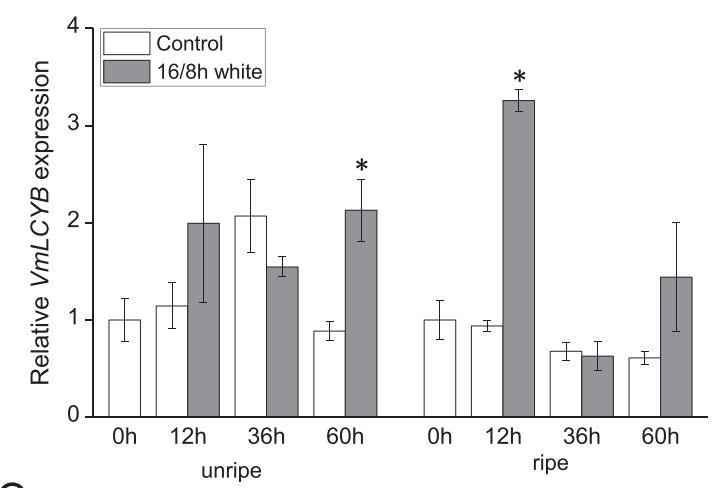

G

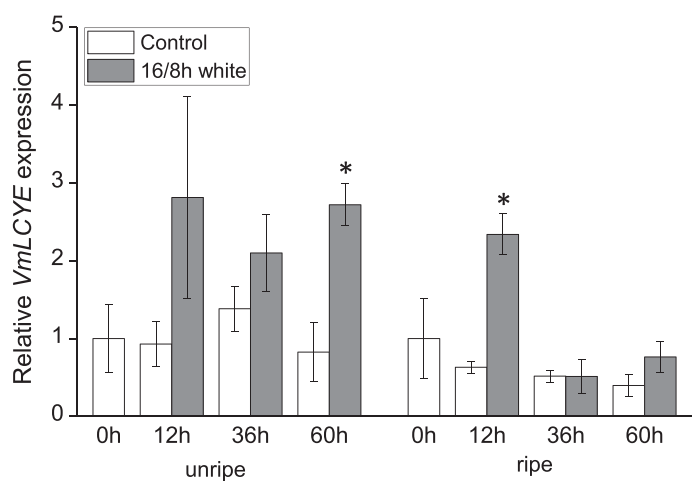

B

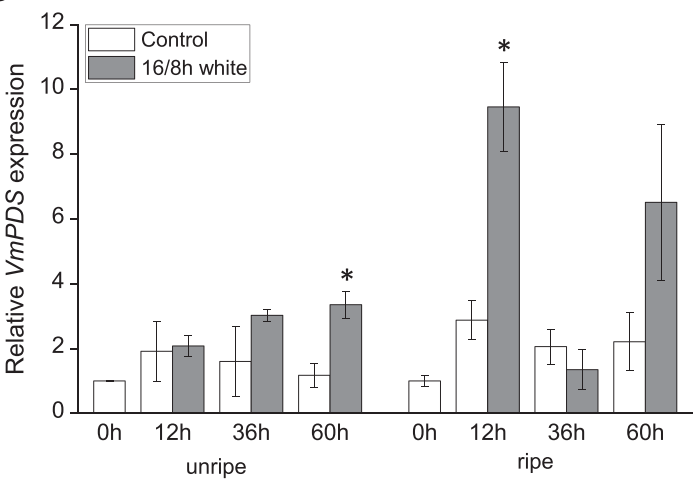

D

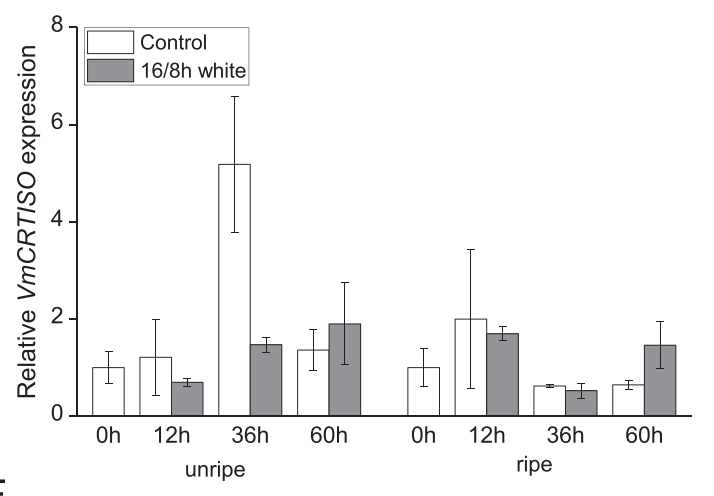

F

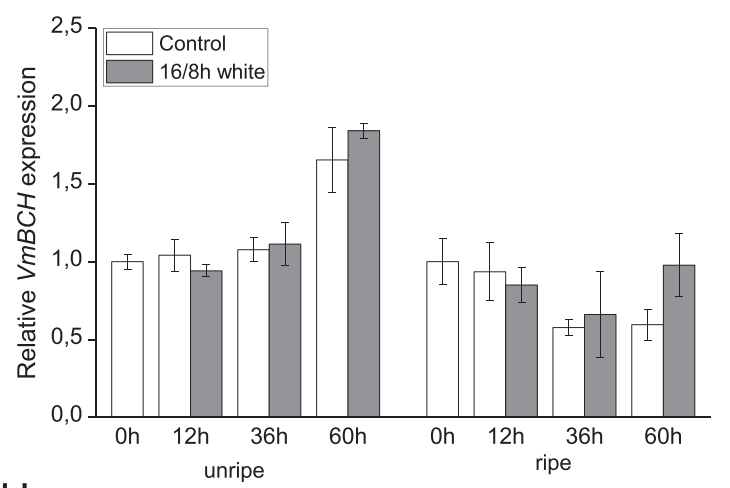

$\mathrm{H}$

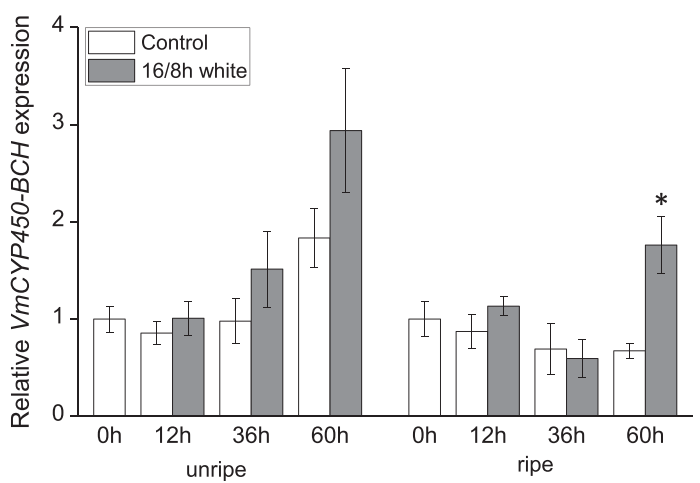

Fig. 5 (See legend on next page.) 
(See figure on previous page.)

Fig. 5 Effect of $16 / 8 \mathrm{~h}$ photoperiodic white light treatment on the expression of carotenoid biosynthetic genes VmPSY (a), VmPDS (b), VmZDS (c), $\operatorname{VmCRTISO}(\mathbf{d}), \operatorname{VmLCYB}(\mathbf{e}), \mathrm{VmBCH}(\mathbf{f}), \mathrm{VmLCYE}(\mathbf{g})$ and $\mathrm{VmCYP450-BCH}(\mathbf{h})$ in unripe (stage S3) and ripe (stage S5) bilberry fruits. The relative expression of the genes was quantified by qRT-PCR and normalized to VmGAPDH. Values represent means \pm SEs of three replicates. Asterisks indicate statistically significant differences from respective control (dark treatment) in Student's $t$-Test $(P \leq 0.05)$

and berries, bilberries can be considered as a good source of carotenoids. Therefore, carotenoids contribute to the overall antioxidant capacity of bilberry fruit in addition to the high anthocyanin [23] and modest ascorbic acid [24] contents.

\section{Carotenoid content during bilberry fruit development is determined by different molecular mechanisms}

In some fruits, such as tomato, Citrus, red-fleshed watermelon (Citrullus lanatus) and sea buckthorn (Hippophae rhamnoides L.), the carotenoid content increases during the fruit maturation indicated by the appearance of yellow to red color in ripening fruit $[14,33,36,37]$. In other fruits, such as strawberry, raspberries, grape and apple, in which the red pigment formation is mostly a consequence of anthocyanin accumulation, a decreasing trend in the carotenoid content over the fruit development has been described [18, 19, 31, 34, 35]. According to the current results, bilberry belongs to the latter group showing a decreasing trend in the content of both carotenes and xanthophylls during the fruit development. The detected decrease in the levels of all carotenoid compounds over the fruit development does not coincide with the notable increment in the expression of the carotenoid biosynthetic genes at the fruit ripening demonstrated in our current study. The up-regulation of the early biosynthetic genes (VmPSY, VmPDS, VmZDS, $V m C R T I S O)$, which generate the flux to carotenoids, as well as the up-regulation of the $V m L C Y B$ with simultaneous down-regulation of the specific genes of the $\varepsilon, \beta$ branch ( $V m L C Y E$ and $V m C Y P 450-B C H)$ at the ripening stage, indicates direction of the carotenoid biosynthesis towards $\beta$-branch at fruit ripening. However, none of the carotenoid compounds in the $\beta$-branch accumulates in the ripening bilberries. This suggests mechanisms beyond transcriptional regulation in the bilberry fruit carotenoid metabolism, and turnover of the carotenoid compounds from the pathway. Although the balance in the expression of the early and late biosynthetic genes is described as a key determinant of the carotenoid profile in many fruits [12-16], also other factors such as post-transcriptional mechanisms and enzymatic degradation of carotenoids to apocarotenoids by CCDs can affect to the final carotenoid content of fruits $[3,6]$. Moreover, xanthophylls can also be found esterified with fatty acids as in the case of lutein esters reported in raspberries [35].

\section{Role of enzymatic degradation in carotenoid content during bilberry fruit ripening}

The CCD1s are cytosolic localized enzymes that have a role in the cleavage of double bonds of carotenoids to form $\mathrm{C}_{13}$ - and $\mathrm{C}_{14}$-apocarotenoids. These enzymes have multiple substrates, including $\mathrm{C}_{27}$-carotenoids in cytosol and $\mathrm{C}_{40}$-carotenoids accessed by $\mathrm{CCD} 1$ in the outer plastid envelope $[38,39]$. In the present study, $V m C C D 1$ showing a high identity to other fruit $C C D 1$ genes was isolated from bilberry fruit and its expression was found to be up-regulated at the onset of bilberry fruit ripening. The high CCD1 expression upon fruit development has earlier been associated with the carotenoid degradation and the formation of apocarotenoids in different fruit species [31, 40-42]. In raspberries, the decrease in the carotenoid content with the parallel increase in the RiCCD1 expression during fruit ripening was suggested to be associated with the exceptionally high accumulation of apocarotenoid aroma volatiles in ripe fruit [18]. Also in grape berries approaching ripening, the increased expression of the $V \nu C C D 1$, cleaving a variety of carotenoid substrates, led to the increased $\mathrm{C}_{13}$-norisoprenoid level in the ripe berries of Muscat of Alexandria and Shiraz cultivars $[29,43]$. On the other hand, in strawberry, the up-regulation of FaCCD1 expression at fruit ripening was suggested to be related with the simultaneous decrease in the carotenoid content, especially lutein [19]. Therefore, in the light of the previous studies concerning CCD1 function in fruits, the detected upregulation of the $V m C C D 1$ in the ripening bilberries, may have a role in decreasing the carotenoid content towards ripe fruit, especially in the $\beta$-branch of the carotenoid pathway.

In our earlier study, we have shown that another wellknown CCD member, VmNCED1, which encodes the key enzyme in the formation of ABA, shows an increase in its expression in the developing bilberry fruit leading to an elevated ABA level at the onset of fruit ripening [11]. This is a similar observation with other nonclimacteric fruits, including strawberry and blueberry $(V$. corymbosum), in which ABA accumulation is considered as an initiator of fruit ripening and anthocyanin production $[44,45]$. The suppression of NCED1 expression blocking the metabolic flux to ABA has been shown to lead to an increased accumulation of upstream carotenoid compounds in tomato [37]. It is possible that the elevated $V m N C E D 1$ expression at fruit ripening [11], which 
A

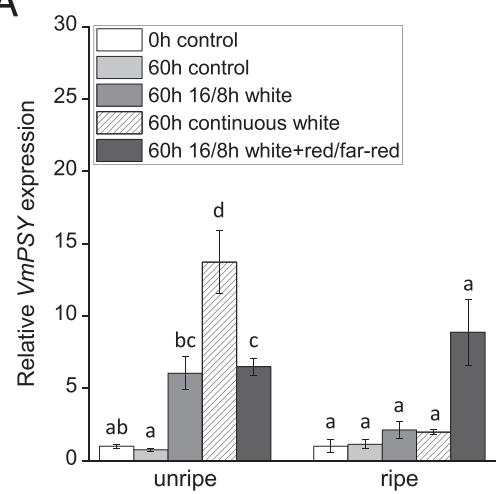

C

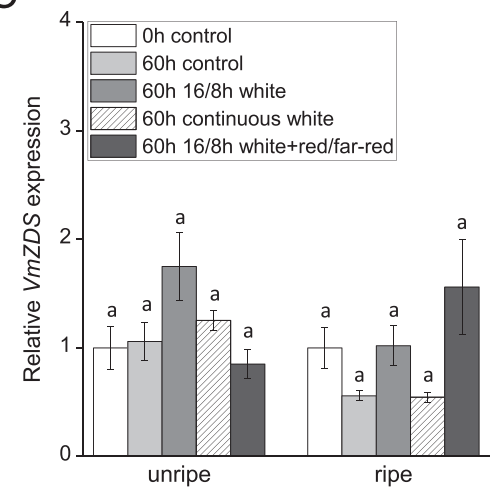

E

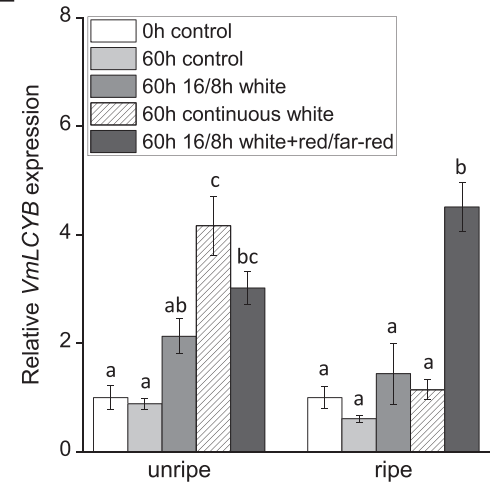

G

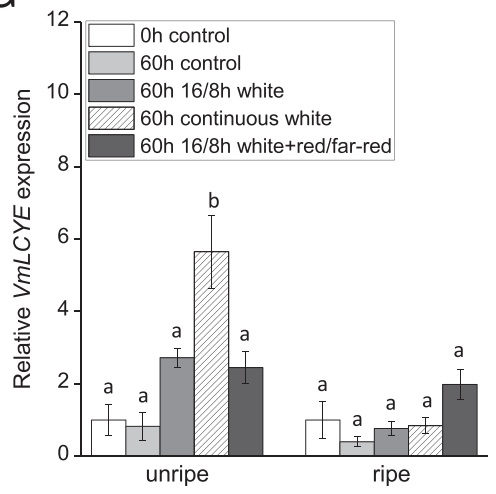

B

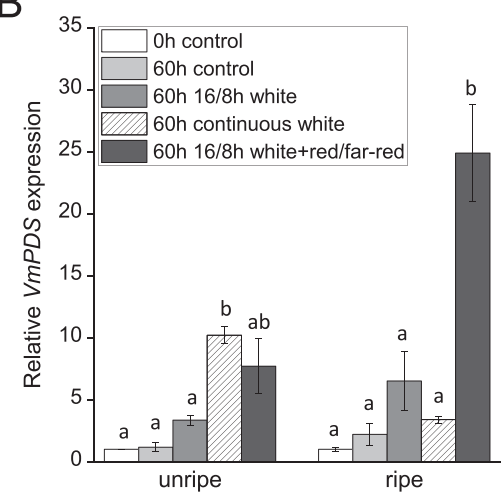

D

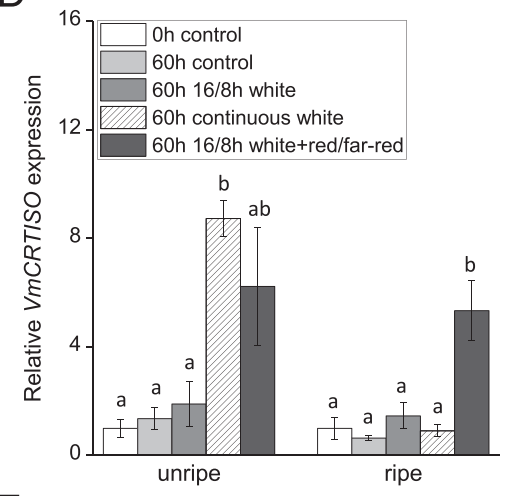

F

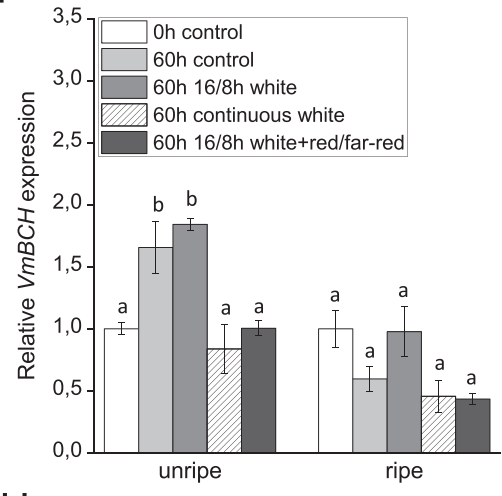

$\mathrm{H}$

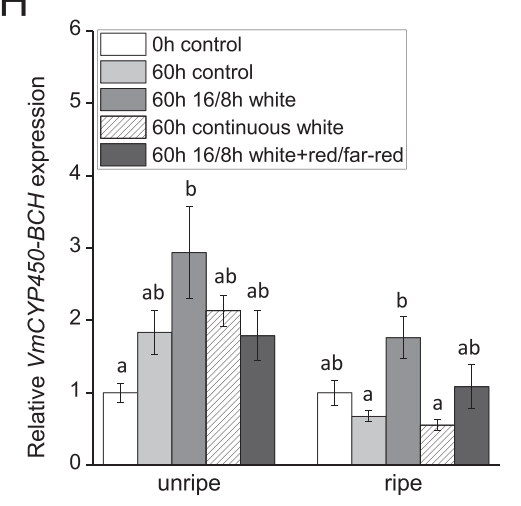

Fig. 6 (See legend on next page.) 
(See figure on previous page.)

Fig. 6 Effect of different light conditions on the expression of carotenoid biosynthetic genes VMPSY (a), VmPDS (b), VmZDS (c), VmCRTISO (d), $\operatorname{VmLCYB}(\mathbf{e}), V m B C H(\mathbf{f}), V m L C Y E(\mathbf{g})$ and $V m C Y P 450-B C H(\mathbf{h})$ in unripe (stage S3) and ripe (stage S5) bilberry fruits. The relative expression of the genes was quantified by qRT-PCR and normalized to VmGAPDH. Values represent means \pm SEs of three replicates. Columns labeled with different letters indicate statistically significant differences ( $P \leq 0.05$, one-way ANOVA with post hoc comparisons)

increases the degradation of violaxanthin and neoxanthin, can affect the carotenoid composition in ripening bilberry fruit. Since anthocyanins are responsible for the pigmentation of ripe bilberry fruits, the transition from photosynthetic green fruit to non-photosynthetic ripening fruit can readily involve degradation and reuse of the carotenoids for the formation of apocarotenoid compounds, such as ABA and volatile aroma compounds, which have been reported in ripe bilberries $[11,46]$. However, other yet unidentified CCD family genes may also be involved in the carotenoid degradation in bilberry.

\section{Light has differential effect on the carotenoid metabolism} between unripe and ripe berries

In addition to the programmed developmental regulation, light conditions seem to influence carotenoid biosynthesis in fruit tissues [21]. The results of the current study show that light up-regulates the expression of the carotenoid pathway genes in the bilberry fruit. The expression of particularly VmPSY, VmPDS, VmCRTISO, and both lycopene cyclases was stimulated by light. Despite of the significant increase in the transcript abundance of the carotenoid biosynthetic genes by all tested $60 \mathrm{~h}$ light treatments, only a slight increase in the carotenoid content in the unripe bilberry fruits was detected after white light treatments in the current study. In ripe berries, the carotenoid content decreased after the light treatments although red wavelengths were shown to increase the expression of the biosynthetic genes (Table 2). This inconsistency between the expression of the biosynthetic genes and the carotenoid content may be attributed to the post-transcriptional regulation or carotenoid degradation. Our current and previous studies [11] suggest the carotenoid degradation to be involved. First, as discussed earlier, the expression of the both $V m C C D 1$ and $V m N C E D 1$ are higher in ripening bilberry fruits compared to the unripe fruits possibly explaining the decrease in the carotenoid content in the ripe fruit. Secondly, the expression of the both $V m C C D 1$ and $V m N C E D 1$ were found in this study also to be upregulated by the light treatments, which could lead to a higher rate of carotenoid breakdown. The up-regulation of the NCED1 expression by light has been reported earlier in the ripening grape berry $[47,48]$ as well as in Citrus where elevated expression of the NCED genes by red light treatment was demonstrated in pulp tissues of three different citrus varieties [49]. Short treatments with white, red or red/far-red light were demonstrated to up-regulate PhCCD1 expression also in dark-adapted Petunia flowers by a phytochrome-independent manner, leading to a high volatile emission [50]. Considering bilberry fruits, our results indicate that despite of the higher flux of metabolites directed to the carotenoid pathway under high light conditions, the carotenoid content decreases due to the increment in the carotenoid cleavage reactions.

Some earlier reports have indicated that light quality and especially red light wavelengths affect the carotenoid biosynthesis in fruits. In post-harvest studies with tomato, red light treatments were demonstrated to increase lycopene content of fruit $[21,51]$. In Citrus, the
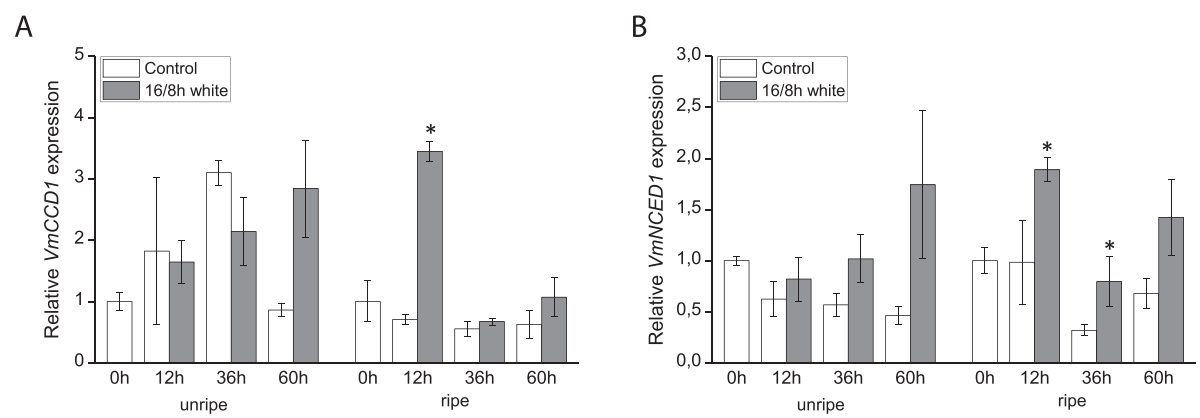

Fig. 7 Effect of 16/8 h photoperiodic white light treatment on the expression of carotenoid cleavage genes VmCCD1 (a) and VmNCED1 (b) in unripe (stage S3) and ripe (stage S5) bilberry fruits. The relative expression of the genes was quantified by qRT-PCR and normalized to VmGAPDH. Values represent means \pm SEs of three replicates. Asterisks indicate statistically significant differences from respective control (dark treatment) in Student's $t$-Test $(P \leq 0.05)$ 

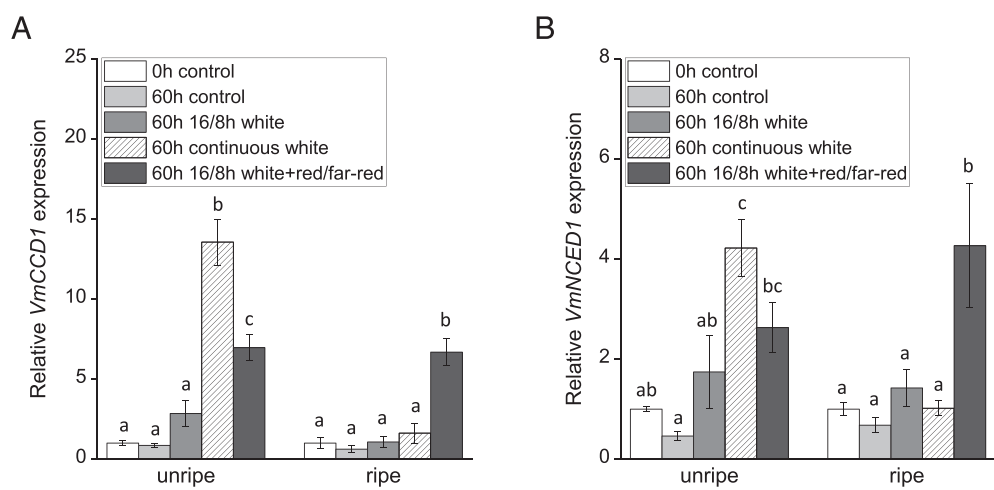

Fig. 8 Effect of different light conditions on the expression of carotenoid cleavage genes VmCCD1 (a) and VmNCED1 (b) in unripe (stage S3) and ripe (stage S5) bilberry fruits. The relative expression of the genes was quantified by qRT-PCR and normalized to VmGAPDH. Values represent means \pm SEs of three replicates. Columns labeled with different letters indicate statistically significant differences $(P \leq 0.05$, one-way ANOVA with post hoc comparisons)

effect of red light treatment on the carotenoid biosynthesis and content was cultivar dependent $[22,49]$. Our results showing the up-regulation of the carotenoid biosynthesis as a response to the red light are in agreement with these studies. Interestingly, we found the effect of the light quality on the expression of both the carotenoid biosynthetic and the cleavage genes to be dependent on fruit developmental stage. In the unripe berries, all the applied $60 \mathrm{~h}$ light treatments induced the expression of the carotenoid biosynthetic and cleavage genes while only red/far-red light wavelengths showed inducing effect in the ripe fruits. The detected differential effect of the light quality on the carotenoid metabolism between the developmental stages can be associated with the differences in the regulation of carotenoid biosynthesis between chloroplasts of unripe fruit and chromoplasts of ripe fruit. Unlike chloroplasts, the carotenoid content and composition in chromoplasts has been shown to vary highly between species and cultivars. It is possible that the red light has a specific role in the metabolism of fruits close to maturity. During tomato fruit ripening, the amount of red light passing through the pericarp tissues was shown to increase four-fold [51]. Therefore, the detected differential effect of the light quality can also be a result of the absorption of certain wavelengths of light by pigments present in the skin of the ripe fruits, such as anthocyanins in the ripe bilberries. Supporting this assumption, it has been shown that anthocyanins strongly absorb wavelengths under $600 \mathrm{~nm}$ in the leaf tissues [52]. The specific role of the red light on the ripening berry tissues and the carotenoid metabolism is supported by the study of Kondo et al. [53] in the grape berries. They showed that especially red light given to the ripening berries induced the expression of the $V v N C E D 1$ and the formation of the plant hormone ABA also leading to the higher anthocyanin concentration. In the non- climacteric grape berries ABA is considered, similarly to Vaccinium berries, as an initiator of the ripening and the anthocyanin biosynthesis.

\section{Conclusions}

This is the first report regarding to the carotenoid metabolism and its regulation in the berries of the genus Vaccinium, which are known for the high anthocyanin and antioxidant activity levels. The results show that among fruits and berries, bilberries are relatively good source of carotenoids, which also contribute to the overall antioxidant activity of the berries. An inconsistency between the carotenoid content and the related biosynthetic gene expression was detected during the bilberry fruit development, indicating a cleavage of carotenoids to apocarotenoids toward ripening of the berries. As a non-climacteric fruit, increase in the ABA formation at the onset of the fruit ripening can contribute to the rapid carotenoid turnover in bilberry. Moreover, light was shown to up-regulate both the carotenoid biosynthetic and the cleavage genes but only red/far-red light wavelengths had a role in the ripe bilberry fruits. Our results indicate that in the ripening bilberries, the red light wavelengths specifically induce the expression of the carotenoid metabolism genes directed to the apocarotenoid formation. Whether this has any effect on the flavor properties of bilberries, requires further investigations. It is also to be studied whether the red light wavelengths can induce the expression of the flavonoid biosynthetic genes in the mature Vaccinium berries through the ABA regulation.

\section{Methods}

Plant material and treatments

All plant material used for the experiments was originated from the natural forest stand in Oulu $\left(65^{\circ} 01^{\prime} \mathrm{N}\right.$, 
Table 2 Effect of light conditions on carotenoid content ( $\mu \mathrm{g} 100 \mathrm{~g}^{-1} \mathrm{DW}$ ) in bilberry fruits

\begin{tabular}{|c|c|c|c|c|c|c|c|c|}
\hline \multirow[t]{2}{*}{ Compound } & \multicolumn{4}{|c|}{ Light treatment for unripe fruits } & \multicolumn{4}{|c|}{ Light treatment for ripe fruits } \\
\hline & Control & 16/8 h white & Continuous white & $16 / 8$ h white + red/far-red & $\overline{\text { Control }}$ & 16/8 h white & Continuous white & 16/8 h white + red/far-red \\
\hline Lutein & $939.44 \pm 95.84$ & $1170.85 \pm 453.17$ & $1221.14 \pm 119.55$ & $892.26 \pm 233.49$ & $969.27 \pm 324.59$ & $457.25 \pm 21.71$ & $564.34 \pm 61.60$ & $503.88 \pm 75.53$ \\
\hline$\beta$-carotene & $296.52 \pm 16.48$ a & $392.43 \pm 137.69 \mathrm{ab}$ & $469.43 \pm 15.71 \mathrm{cb}$ & $265.46 \pm 90.87 \mathrm{ab}$ & $342.62 \pm 53.00$ & $294.33 \pm 8.92$ & $251.40 \pm 12.38$ & $302.11 \pm 15.34$ \\
\hline 9Z- $\beta$-carotene & $140.30 \pm 6.57$ & $158.06 \pm 36.20$ & $159.82 \pm 2.90$ & $141.29 \pm 15.52$ & $148.86 \pm 8.42$ & $140.74 \pm 2.74$ & $141.51 \pm 4.22$ & $141.90 \pm 2.27$ \\
\hline Zeaxanthin & ud & $162.76 \pm 16.74$ & $185.18 \pm 5.22$ & $187.94 \pm 18.35$ & ud & ud & ud & ud \\
\hline Antheraxanthin & $67.37 \pm 5.35$ & $84.91 \pm 15.01$ & $69.15 \pm 0.85$ & $70.55 \pm 8.23$ & $69.29 \pm 15.33$ & ud & $54.65 \pm 1.22$ & ud \\
\hline Violaxanthin & $356.08 \pm 29.29$ & $409.93 \pm 115.15$ & ud & $291.98 \pm 13.36$ & $425.91 \pm 62.40$ & $320.89 \pm 17.33$ & $324.18 \pm 24.68$ & $306.41 \pm 4.82$ \\
\hline Neoxanthin & $272.28 \pm 51.13$ & $356.33 \pm 33.22$ & $310.67 \pm 67.01$ & $301.75 \pm 67.64$ & $228.60 \pm 41.17$ & $154.95 \pm 4.05$ & $187.90 \pm 5.48$ & $179.80 \pm 11.63$ \\
\hline$\beta$-cryptoxanthin & ud & ud & ud & ud & ud & ud & $128.00 \pm 90.51$ & $132.33 \pm 76.40$ \\
\hline Total carotenoids & $2071.99 \pm 140.58$ & $2681.03 \pm 703.42$ & $2508.56 \pm 241.57$ & $1979.33 \pm 547.75$ & $2163.68 \pm 485.14$ & $1368.16 \pm 46.20$ & $1505.76 \pm 82.74$ & $1451.98 \pm 107.76$ \\
\hline
\end{tabular}

Values represent means \pm SEs of three replicates

Only statistically significant differences are presented by different letters ( $P \leq 0.05$, one-way ANOVA with post hoc comparisons)

DW dry weight, ud under detection limit 
$25^{\circ} 28^{\prime}$ E), Finland. All material sampling was conducted complying with national guidelines. Five developmental stages of bilberry (Vaccinium myrtillus L.) fruit (Fig. 1b) were collected from June to August 2011. The developmental stages of the fruits were S1, flower collected June $7^{\text {th }}$ (anthesis); S2, small unripe green fruit (15 days after anthesis); S3, large unripe green fruit just before coloring began (28 days after anthesis); S4, ripening purple fruit (34 days after anthesis) and S5, fully ripe blue fruit (55 days after anthesis) as described earlier [11]. Immediately after collection, all samples were frozen in liquid nitrogen and stored at $-80{ }^{\circ} \mathrm{C}$ until they were used for RNA extraction and determination of carotenoids.

In order to study the effect of the light conditions on the carotenoid metabolism, different light treatments were applied to the bilberry plants holding fruits: photoperiodic 16/ $8 \mathrm{~h}$ (light/dark) white light, continuous white light (24 h) and photoperiodic 16/8 h (light/dark) white light enhanced with red and far-red light wavelengths. Control plants were kept in the constant darkness. Light treatments were applied to the three replicate collections of bilberries at two different stages of fruit development; either on unripe S3 stage (collected at June $13^{\text {th }}, 2013$ ) or mature S5 stage (collected at July $3^{\text {rd }}, 2013$ ). In each separate experiment, bilberries were initially kept in the darkness for $24 \mathrm{~h}$ before exposed for five days to the different light treatments. During the light treatments, bilberries were placed under VP3411 M16 -light emitting diode (LED) lamps (Valopaa, Oulu, Finland) and irradiated with continuous or photoperiodic white light (400-800 nm; Additional file 1). The additional red to far-red light $(600-800 \mathrm{~nm})$ was provided combining the VP3411 M16 -LED lamps with Ecoline R7s halogen lamps (Osram, Vantaa, Finland) filtrated by the Lucite Abmer 300 transparent plex (Additional file 1). The light intensity, measured by the USB RAD+ spectroradiometer (Ocean Optics Inc., Dunedin, FL, USA), was $152 \mu \mathrm{mol} \mathrm{m} \mathrm{m}^{-2} \mathrm{~s}^{-1}$ under the white light treatment and $200 \mu \mathrm{mol} \mathrm{m} \mathrm{m}^{-2} \mathrm{~s}^{-1}$ under the white + red/far-red light treatment. The increase in the light intensity in white $+\mathrm{red} / \mathrm{far}-$ red light treatment was solely due to the increase in the red to far-red light wavelengths (Additional file 1). In the white light treatments, the red/far-red light ratio was 8.9 and after the supplementation of the white light with red to far-red light the ratio was 1.5. Temperature in the growth room was maintained $20-21{ }^{\circ} \mathrm{C}$ throughout the experiments. Berry samples were collected after $0,12,36$ and $60 \mathrm{~h}$ from the beginning of the light treatments for the gene expression analyses and at day $5(116 \mathrm{~h})$ for the analyses of carotenoids. Immediately after the excision, all berry samples were frozen in liquid nitrogen and stored at $-80{ }^{\circ} \mathrm{C}$ until they were used for RNA extraction and determination of carotenoids. The plant material was identified by Dr. Marian Sarala and voucher samples are stored at the Genetics and Physiology Unit, University of Oulu, Finland.

\section{Isolation of RNA and CDNA preparation}

Total RNA was isolated from the samples according to the method described for bilberry [54]. The cDNA was synthesized from the total RNA using SuperScript III reverse transcriptase (Invitrogen, Carlsbad, CA, USA) according to the manufacturer's instructions. The cDNA was purified from the contaminating genomic DNA by using the method described by Jaakola et al. [55].

\section{Isolation of carotenoid biosynthetic genes}

The amplification of the sequences of the key carotenoid biosynthetic genes, phytoene synthase (VmPSY), phytoene desaturase $(V m P D S)$, $\zeta$-carotene desaturase $(V m Z D S)$, carotenoid isomerase (VmCRTISO), lycopene $\beta$-cyclase $(V m L C Y B)$, lycopene $\varepsilon$-cyclase $(V m L C Y E), \beta$-carotene hydroxylase $(\mathrm{VmBCH})$ and carotenoid $\beta$-ring hydroxylase of cytochrome P450 family ( $\mathrm{VmCYP450-BCH)}$ as well as the carotenoid cleavage dioxygenase class 1 ( $V m C C D 1)$, was achieved from berry cDNA with gene-specific primers that were designed based on the sequences of the respective genes found in $V$. corymbosum transcriptome database [56]. The PCR-reactions were performed by using DyNazyme $^{\text {tu }}$ II DNA polymerase (Finnzymes, Espoo, Finland). The amplified PCR products were gel-purified using Montage $e^{\circ}$ DNA Gel Extraction Kit (Millipore, Bedford, MA, USA). The purified PCR products were ligated into a pGEM-T Easy vector (Promega, Madison, WI, USA) and sequenced using an ABI 3730 DNA sequencer (Applied Biosystems, Foster City, CA, USA) with a BigDye Terminator Cycle Sequencing Kit (Applied Biosystems).

\section{Relative quantification of gene expression}

Real-time quantitative reverse transcription PCR (qRTPCR) analyses were performed with a LightCycler 480 instrument and software (Roche Applied Sciences, Indianapolis, IN, USA). The transcript abundance of the bilberry carotenoid biosynthetic genes (VmPSY, VmPDS, VmZDS, VmCRTISO, VmLCYB, VmLCYE, VmBCH, $V m C Y P 450-B C H)$ and the cleavage genes (VmCCD1, VmNCED1; GenBank accession no. JX982599) was detected using a LightCycler ${ }^{\circ}$ SYBR Green I Master qPCR kit (Roche). The qRT-PCR conditions were an initial incubation at $95{ }^{\circ} \mathrm{C}$ for $10 \mathrm{~min}$ followed by 45 cycles of $95{ }^{\circ} \mathrm{C}$ for $10 \mathrm{~s}, 60{ }^{\circ} \mathrm{C}$ for $20 \mathrm{~s}$, and $72{ }^{\circ} \mathrm{C}$ for $10 \mathrm{~s}$. The gene-specific primer sequences used for the qRT-PCR analysis are shown in Table 3. Glyceraldehyde-3phosphate dehydrogenase (VmGAPDH; GenBank accession no. AY123769) was used as a reference gene for the relative quantification of the PCR products. The results were calculated with LightCycler $^{\circ} 480$ software (Roche), using the calibrator-normalized PCR efficiency-corrected method (Technical note no. LC 13/2001, Roche). The amplification of only one product in qRT-PCR was confirmed by a melting curve analysis and sequencing. 
Table 3 Gene-specific primers used for qRT-PCR analysis

\begin{tabular}{|c|c|}
\hline Gene & Primer sequence $5^{\prime}-3^{\prime}$ \\
\hline \multirow[t]{2}{*}{ VMPSY } & ATGCATCGCACATAACTCCA (forward) \\
\hline & GGTCCATCCTCATTCCTTCA (reverse) \\
\hline \multirow[t]{2}{*}{ VmPDS } & GTTGCAGCGGAAAGAACATT (forward) \\
\hline & CATTGCTGGCAGTAGTCCAA (reverse) \\
\hline \multirow[t]{2}{*}{ VmZDS } & TGCCATTACCAAATGACGAA (forward) \\
\hline & ATATGAGCCAGCGAGGAAGA (reverse) \\
\hline \multirow[t]{2}{*}{ VmCRTISO } & TCTTGAGTGCTTGACGCTTG (forward) \\
\hline & GGGTAGTTGATTCCCCCAAA (reverse) \\
\hline \multirow[t]{2}{*}{$V m L C Y B$} & ACGGCGTTAAGTTCCATCAA (forward) \\
\hline & TCAGCCAAAATCCCATAAGC (reverse) \\
\hline \multirow[t]{2}{*}{ VmLCYE } & TCGTTCTTACGGGCGAGTTA (forward) \\
\hline & TTCCAGAAGCTGCTCCAGAT (reverse) \\
\hline \multirow[t]{2}{*}{$\mathrm{VmBCH}$} & TATCGGAGATGTTTGGCACA (forward) \\
\hline & ACCGCGTTATAATGGCAAA (reverse) \\
\hline \multirow[t]{2}{*}{ VmCYP450-BCH } & ATGTGTTGGCGACATGTTG (forward) \\
\hline & GCATCTCCAATTCAGGCAGT (reverse) \\
\hline \multirow[t]{2}{*}{ VmCCD1 } & ATGCTGAGAGCAAGGCTGAAAG (forward) \\
\hline & ATCCAACATGCCAAGAGTCTGC (reverse) \\
\hline \multirow[t]{2}{*}{ VmNCED1 } & CCCGAACAGGGGAGGATATT (forward) \\
\hline & CGGTCAACACGGACTTCAAA (reverse) \\
\hline \multirow[t]{2}{*}{ VmGAPDH } & CAAACTGTCTTGCCCCACTT (forward) \\
\hline & CAGGCAACACCTTACCAACA (reverse) \\
\hline
\end{tabular}

\section{Analysis of carotenoids}

Carotenoids were extracted using the method described earlier [35] with small modifications. Freeze-dried flowers and berries were ground into a fine powder under liquid nitrogen using a mortar and pestle. Fifty milligrams of each sample was mixed with $6 \%$ methanolic $\mathrm{KOH}$, vortexed vigorously and incubated for $2.5 \mathrm{~h}$ at room temperature. Carotenoids were extracted twice with $1 \mathrm{ml}$ of hexane and the extracts were dried by centrifugal evaporation. The dried residues were stored at $-20{ }^{\circ} \mathrm{C}$ before dissolved in $50 \mu \mathrm{l}$ ethyl-acetate for HPLC. All steps were carried out, when possible, on ice and shielded from light to prevent carotenoid degradation.

HPLC-analysis was performed according to Fraser et al. [57] by using a 1290 Agilent UPLC equipped with a reverse-phase $C_{30}$ column $(250 \times 2.1 \mathrm{~mm})$ with $3 \mu \mathrm{m}$ particle size coupled to a $C_{30} 20 \times 4.6 \mathrm{~mm}$ guard column (YMC Inc., Wilmington, NC, USA). The mobile phases consisted of methanol (A) and tert-methyl butyl ether containing $5 \%$ of water/methanol $(20 / 80 \mathrm{v} / \mathrm{v})$ and $0.2 \%(\mathrm{w} / \mathrm{v})$ ammonium acetate (B). The gradient elution used was $100 \%$ A, $0 \%$ B isocratically for the initial $6 \mathrm{~min}$, a step to $82.5 \% \mathrm{~A}$ and $17.5 \% \mathrm{~B}$ at $7 \mathrm{~min}$ maintained for $5 \mathrm{~min}$, followed by a linear gradient to $32.5 \% \mathrm{~A}$ and $67.5 \% \mathrm{~B}$ by
32 min, which was maintained for $14 \mathrm{~min}$. A conditioning phase (48-60 min) was used to return the column to the initial concentrations of $\mathrm{A}$ and $\mathrm{B}$. Flow rates of $0.21 \mathrm{ml} \mathrm{min}$ ${ }^{-1}$ were used and injection volume was $3 \mu \mathrm{l}$. The DAD (diode array detector) signal was acquired monitoring the eluate continuously from $200 \mathrm{~nm}$ to $600 \mathrm{~nm}$. Carotenes and xanthophylls were quantified considering the area of each peak at a wavelength of $450 \mathrm{~nm}$. Quantification of individual compounds was performed by external standard calibration curves by using the respective standards and the script developed by Wehrens et al. [58].

\section{Statistical analysis}

Quantitative results of the gene expression and metabolite analyses are presented in terms of means \pm SEs of at least three biological replicates. The effect of light conditions on the gene expression and the carotenoid content were analysed by either Student's $t$-Test or one-way analysis of variance (ANOVA) followed by Tukey's HSD test (or in cases where the homogeneity of variances assumption was not met, the Games-Howell test) using SPSS Statistics program, version 22 (IBM, New York, NY, USA).

\section{Ethics and consent to participate \\ Not applicable.}

\section{Consent to publish}

Not applicable.

\section{Availability of data and materials}

The data sets supporting the results of this article are included within the article and its additional files. All the obtained sequences from bilberry were deposited to the GenBank database and the accession numbers are shown in Table 1.

\section{Additional file}

Additional file 1: Spectra of light treatments applied to bilberry fruits. (PDF $27 \mathrm{~kb}$ )

\section{Abbreviations \\ ABA: abscisic acid; $\mathrm{BCH}$ : $\beta$-carotene hydroxylase; $C \mathrm{CD}$ : carotenoid cleavage dioxygenase; CRTISO: carotenoid isomerase; CYP450-BCH: carotenoid $\beta$-ring hydroxylase of cytochrome P450 family; LCYB: lycopene $\beta$-cyclase; LCYE: lycopene $\varepsilon$-cyclase; NCED: 9-cis-epoxycarotenoid dioxygenase; PDS: phytoene desaturase; PSY: phytoene synthase; qRT-PCR: real-time quantitative reverse transcription PCR; ZDS: ל-carotene desaturase.}

\section{Competing interests}

The authors declare that they have no competing interests.

\section{Authors' contributions}

Experiments were designed by KK, MS and LJ. Expression analyses were performed by KK, MS, JH and HM. Metabolic analyses were performed by LZ, EC and SM. KK, LZ and $\amalg$ were responsible of manuscript writing with contribution of MS, HH, SM and EC. All authors read and approved the final manuscript. 


\section{Acknowledgements}

We thank Matti Rauman for contribution in setting up the light systems. We thank also Domenico Masuero (Edmund Mach Foundation) for the help with carotenoid analysis.

\section{Funding}

The present study was financially supported by Kone Foundation (to $L$ ), The STSM (Short Term Scientific Program) program within the COST action FA1006 (PlantEngine) and the Finnish Doctoral Program in Plant Science (to LZ).

\section{Author details}

${ }^{1}$ Genetics and Physiology Unit, University of Oulu, P.O. Box 3000 FI-90014 Oulu, Finland. ${ }^{2}$ Climate laboratory Holt, Department of Arctic and Marine Biology, UiT the Arctic University of Norway, NO-9037 Tromsø, Norway. ${ }^{3}$ Fondazione Edmund Mach, Research and Innovation Center, via E. Mach 1 , 38010, San Michele all'Adige, TN, Italy. ${ }^{4} \mathrm{NIBIO}$, Norwegian Institute of Bioeconomy Research, P.O. Box 115 NO-1431 Ås, Norway.

\section{Received: 2 February 2016 Accepted: 14 April 2016 Published online: 21 April 2016}

\section{References}

1. Jimenez-Garcia SN, Guevara-Gonzalez RG, Miranda-Lopez R, Feregrino-Perez AA, Torres-Pacheco I, Vazquez-Cruz MA. Functional properties and quality characteristics of bioactive compounds in berries: Biochemistry, biotechnology, and genomics. Food Res Int. 2013;54:1195-207.

2. Nile SH, Park SW. Edible berries: Bioactive components and their effect on human health. Nutrition. 2014;30:134-44.

3. Cazzonelli Cl, Pogson BJ. Source to sink: regulation of carotenoid biosynthesis in plants. Trends Plant Sci. 2010;15:266-74.

4. Vogel JT, Tieman DM, Sims CA, Odabasi AZ, Clark DG, Klee HJ. Carotenoid content impacts flavor acceptability in tomato (Solanum lycopersicum) J Sci Food Agric. 2010;90:2233-40.

5. Liu L, Shao Z, Zhang M, Wang Q. Regulation of carotenoid metabolism in tomato. Mol Plant. 2015;8:28-39.

6. McQuinn RP, Giovannoni JJ, Pogson BJ. More than meets the eye: from carotenoid biosynthesis, to new insights into apocarotenoid signaling. Curr Opinion Plant Biol. 2015;27:172-9.

7. Maiani G, Periago Castón MJ, Catasta G, Toti E, Cambrodón IG, Bysted A, et al. Carotenoids: Actual knowledge on food sources, intakes, stability and bioavailability and their protective role in humans. Mol Nutr Food Res. 2009; 53:S194-218.

8. Fernández-Carcía E, Carvajal-Lérida I, Jarén-Galán M, Garrido-Fernández J, Pérez-Gálvez A, Hornero-Méndez D. Carotenoids bioavailability from foods: From plant pigments to efficient biological activities. Food Res Int. 2012;46: 438-50

9. Ma L, Lin XM. Effects of lutein and zeaxanthin on aspects of eye health. J Sci Food Agric. 2010;90:2-12.

10. Stigliani AL, Giorio G, D'Ambrosio C. Characterization of P450 carotenoid $\beta$ and $\varepsilon$-hydroxylases of tomato and transcriptional regulation of xanthophyll biosynthesis in root, leaf, petal and fruit. Plant Cell Physiol. 2011;52:851-65.

11. Karppinen K, Hirvelä E, Nevala T, Sipari N, Suokas M, Jaakola L. Changes in the abscisic acid levels and related gene expression during fruit development and ripening in bilberry (Vaccinium myrtillus L.). Phytochemistry. 2013;95:127-34.

12. Kato M, Ikoma Y, Matsumoto H, Sugiura M, Hyodo H, Yano M. Accumulation of carotenoids and expression of carotenoid biosynthetic genes during maturation in citrus fruit. Plant Physiol. 2004;134:824-37.

13. Kita M, Kato M, Ban Y, Honda C, Yaegaki H, Ikoma Y, et al. Carotenoid accumulation in Japanise apricot (Prunus mume Siebold \& Zucc.): molecular analysis of carotenogenic gene expression and ethylene regulation. J Agric Food Chem. 2007;55:3414-20.

14. Alquezar B, Rodrigo MJ, Zacarías L. Regulation of carotenoid biosynthesis during fruit maturation in the red-fleshed orange mutant Cara Cara. Phytochemistry. 2008;69:1997-2007.

15. Devitt LC, Fanning K, Dietzgen RG, Holton TA. Isolation and functional characterization of a lycopene $\beta$-cyclase gene that controls fruit colour of papaya (Carica papaya L.). J Exp Bot. 2010;61:33-9.

16. Bramley PM. Regulation of carotenoid formation during tomato fruit ripening and development. J Exp Bot. 2002;53:2107-13.
17. Grassi S, Piro G, Lee JM, Zheng Y, Fei Z, Dalessandro G, et al. Comparative genomics reveals candidate carotenoid pathway regulators of ripening watermelon fruit. BMC Genomics. 2013;14:781.

18. Beekwilder J, van der Meer IM, Simic A, Uitdewilligen J, van Arkel J, de Vos $\mathrm{RCH}$, et al. Metabolism of carotenoids and apocarotenoids during ripening of raspberry fruit. BioFactors. 2008;34:57-66.

19. García-Limones C, Schnäbele K, Blanco-Portales R, Bellido ML, Caballero JL, Schwab W, et al. Functional characterization of FaCCD1: A carotenoid cleavage dioxygenase from strawberry involved in lutein degradation during fruit ripening. J Agric Food Chem. 2008;56:9277-85.

20. Mendes-Pinto MM. Carotenoid breakdown products the - norisoprenoids in wine aroma. Arch Biochem Biophys. 2009;483:236-45.

21. Liu LH, Zabaras D, Bennett LE, Aguas P, Woonton BW. Effects of UV-C, red light and sun light on the carotenoid content and physical qualities of tomatoes during post-harvest storage. Food Chem. 2009;115:495-500.

22. Ma G, Zhang L, Kato M, Yamawaki K, Kiriiwa Y, Yahata M, et al. Effect of blue and red LED light irradiation on $\beta$-cryptoxanthin accumulation in the flavedo of Citrus fruits. J Agric Food Chem. 2012;60:197-201.

23. Zoratti L, Sarala M, Carvalho E, Karppinen K, Martens S, Giongo L, et al. Monochromatic light increases anthocyanin content during fruit development in bilberry. BMC Plant Biol. 2014;14:377.

24. Cocetta G, Karppinen K, Suokas M, Hohtola A, Häggman H, Spinardi A, et al. Ascorbic acid metabolism during bilberry (Vaccinium myrtillus L.) fruit development. J Plant Physiol. 2012;169:1059-65.

25. Heinonen MI, Ollilainen V, Linkola EK, Varo PT, Koivistoinen PE. Carotenoids in Finnish foods: vegetables, fruits, and berries. J Agric Food Chem. 1989;37: 655-9.

26. Marinova D, Ribarova F. HPLC determination of carotenoids in Bulgarian berries. J Food Comp Anal. 2007;20:370-4.

27. Bunea A, Rugină D, Pintea A, Andrei S, Bunea C, Pop R, et al. Carotenoid and fatty acid profiles of bilberries and cultivated blueberries from Romania. Chem Pap. 2012;66:935-9.

28. Lashmanova KA, Kuzivanova OA, Dymova OV. Northern berries as a source of carotenoids. Acta Biochim Pol. 2012;59:133-4.

29. Mathieu S, Terrier N, Procureur J, Bigey F, Günata Z. A carotenoid cleavage dioxygenase from Vitis vinifera $\mathrm{L}$. f functional characterization and expression during grape berry development in relation to $C_{13}$-norisoprenoid accumulation. J Exp Bot. 2005;56:2721-31.

30. Fanciullino AL, Cercós M, Dhuique-Mayer C, Froelicher Y, Talón M, Ollitrault $P$, et al. Changes in carotenoid content and biosynthetic gene expression in juice sacs of four orange varieties (Citrus sinensis) differing in flesh fruit color. J Agric Food Chem. 2008;56:3628-38.

31. Ampomah-Dwamena C, Dejnoprat S, Lewis D, Sutherland P, Volz RK, Allan AC. Metabolic and gene expression analysis of apple (Malus $\times$ domestica) carotenogenesis. J Exp Bot. 2012;63:4497-511.

32. Rodrigues-Uribe L, Guzman I, Rajapakse W, Richins RD, O'Connell MA. Carotenoid accumulation in orange-pigmented Capsicum annuum fruit, regulated at multiple levels. J Exp Bot. 2012;63:517-26.

33. Lv P, Li N, Liu H, Gu H, Zhao W. Changes in carotenoid profiles and in the expression pattern of the genes in carotenoid metabolisms during fruit development and ripening in four watermelon cultivars. Food Chem. 2015; 174:52-9.

34. Young PR, Lashbrooke JG, Alexandersson E, Jacobson D, Moser C, Velasco R, et al. The genes and enzymes of the carotenoid metabolic pathway in Vitis vinifera L. BMC Genomics. 2012;13:243.

35. Carvalho E, Fraser PD, Martens S. Carotenoids and tocopherols in yellow and red raspberries. Food Chem. 2013;139:744-52.

36. Andersson SC, Olsson ME, Johansson E, Rumpunen K. Carotenoids in sea buckthorn (Hippophae rhamnoides L.) berries during ripening and use of pheophytin $a$ as a maturity marker. J Agric Food Chem. 2009;57:250-8.

37. Sun L, Yuan B, Zhang M, Wang L, Cui M, Wang Q, et al. Fruit-specific RNAimediated suppression of SINCED1 increases both lycopene and $\beta$-carotene contents in tomato fruit. J Exp Bot. 2012;63:3097-108.

38. Floss DS, Walter MH. Role of carotenoid cleavage dioxygenase 1 (CCD1) in apocarotenoid biogenesis revisited. Plant Signal Behav. 2009;4:172-5.

39. Gonzales-Jorge S, Ha SH, Magallanes-Lundback M, Gilliland LU, Zhou A, Lipka AE, et al. CAROTENOID CLEAVAGE DIOXYGENASE4 is a negative regulator of $\beta$-carotene content in Arabidopsis seeds. Plant Cell. 2013;25: $4812-26$.

40. Simkin AJ, Schwartz SH, Auldridge M, Taylor MG, Klee HJ. The tomato carotenoid cleavage dioxygenase 1 genes contribute to the formation of the 
flavor volatiles $\beta$-ionone, pseudoionone, and geranylacetone. Plant J. 2004; 40:882-92.

41. Ibdah M, Azulay Y, Portnoy V, Wasserman B, Bar E, Meir A, et al. Functional characterization of $C m C C D 1$, a carotenoid cleavage dioxygenase from melon. Phytochemistry. 2006;67:1579-89.

42. Kato M, Matsumoto H, Ikoma Y, Okuda H, Yano M. The role of carotenoid cleavage dioxygenases in the regulation of carotenoid profiles during maturation in citrus fruit. J Exp Bot. 2006;57:2153-64.

43. Lashbrooke JG, Young PR, Dockrall SJ, Vasanth K, Vivier MA. Functional characterisation of three members of the Vitis vinifera $\mathrm{L}$. carotenoid cleavage dioxygenase gene family. BMC Plant Biol. 2013;13:156.

44. Jia HF, Chai YM, Li CL, Lu D, Luo JJ, Qin L, et al. Abscisic acid plays an important role in the regulation of strawberry fruit ripening. Plant Physiol. 2011;157:188-99

45. Zifkin M, Jin A, Ozga JA, Zaharia LI, Schernthaner JP, Gesell A, et al. Gene expression and metabolite profiling of developing highbush blueberry fruit indicates transcriptional regulation of flavonoid metabolism and activation of abscisic acid metabolism. Plant Physiol. 2012;158:200-24.

46. Rohloff J, Nestby R, Nes A, Martinussen I. Volatile profiles of European blueberry: few major players, but complex aroma patterns. Latvian J Agron. 2009;12:98-103.

47. Azuma A, Yakushiji H, Koshita Y, Kobayashi S. Flavonoid biosynthesis-related genes in grape skin are differentially regulated by temperature and light conditions. Planta. 2012;236:1067-80.

48. Guan L, Dai Z, Wu BH, Wu J, Merlin I, Hilbert G, et al. Anthocyanin biosynthesis is differentially regulated by light in the skin and flesh of white-fleshed and teinturier grape berries. Planta. 2016;243:23-41.

49. Zhang L, Ma G, Kato M, Yamawaki K, Takagi T, Kiriiwa Y, et al. Regulation of carotenoid accumulation and the expression of carotenoid metabolic genes in citrus juice sacs in vitro. J Exp Bot. 2012;63:871-86.

50. Simkin AJ, Underwood BA, Auldridge M, Loucas HM, Shibuya K, Schmelz E, et al. Circadian regulation of the PhCCD1 carotenoid cleavage dioxygenase controls emission of $\beta$-ionone, a fragrance volatile of petunia flowers. Plant Physiol. 2004;136:3504-14.

51. Alba R, Cordonnier-Pratt MM, Pratt LH. Fruit-localized phytochromes regulate lycopene accumulation independently of ethylene production in tomato. Plant Physiol. 2000:123:363-70.

52. Merzlyak MN, Chivkunova OB, Solovchenko AE, Naqvi KR. Light absorption by anthocyanins in juvenile, stressed, and senescing leaves. J Exp Bot. 2008; 59:3903-11.

53. Kondo S, Tomiyama H, Rodyoung A, Okawa K, Ohara H, Sugaya S, et al. Abscisic acid metabolism and anthocyanin synthesis in grape skin are affected by light emitting diode (LED) irradiation at night. J Plant Physiol. 2014:171:823-9.

54. Jaakola L, Pirttilä AM, Halonen M, Hohtola A. Isolation of high quality RNA from bilberry (Vaccinium myrtillus L.) fruit. Mol Biotechnol. 2001:19:201-3.

55. Jaakola L, Pirttilä AM, Vuosku J, Hohtola A. Method based on electrophoresis and gel extraction for obtaining genomic DNA-free CDNA without DNase treatment. BioTechniques. 2004;37:744-8.

56. Rowland L, Alkharouf N, Darwish O, Ogden EL, Polashock JJ, Bassil NV, et al. Generation and analysis of blueberry transcriptome sequences from leaves, developing fruit, and flower buds from cold acclimation through deacclimation. BMC Plant Biol. 2012;12:46.

57. Fraser PD, Pinto MES, Holloway DE, Bramley PM. Application of highperformance liquid chromatography with photodiode array detection to the metabolic profiling of plant isoprenoids. Plant J. 2000;24:551-8.

58. Wehrens R, Carvalho E, Masuero D, de Juan A, Martens S. High-throughput carotenoid profiling using multivariate curve resolution. Anal Bioanal Chem. 2013;405:5075-86.

\section{Submit your next manuscript to BioMed Central and we will help you at every step:}

- We accept pre-submission inquiries

- Our selector tool helps you to find the most relevant journal

- We provide round the clock customer support

- Convenient online submission

- Thorough peer review

- Inclusion in PubMed and all major indexing services

- Maximum visibility for your research

Submit your manuscript at www.biomedcentral.com/submit
Biomed Central 